\title{
Performance Evaluation and Comparison of PDTMRP and MAODV
}

\author{
Megha B Raule \\ MBES College of Engineering Ambajogai, \\ Maharashtra, India
}

\author{
Bankat M. Patil. \\ MBES College of Engineering Ambajogai, \\ Maharashtra, India
}

\begin{abstract}
A mobile host don't have fixed infrastructure in Mobile ad hoc network. And, each mobile host is interconnected with another host through wireless network. The portable host has multi-jump transmission ability and it needs to serve as a switch. The mobile host owing a dynamic topology and resource and routing scheme in MANET presents an important challenge. A force mindful double tree based multicast directing convention (PDTMRP) for MANET's is compared with MAODV. All node in networks are randomly classified into two types,group-1 \& group-2.To achieve the load balance in network, we have constructed two multicast trees into two group like tree-1 for group-1 \&tree-2 for group-2.The simulation results show that the PDTMRP schemes performs more better than multicast specially appointed on interest separation vector directing convention (MAODV). Thus PDTMRP system outperform in terms of Performance assessment measurements, for example, parcel conveyance proportion, control overhead, packet delivery delay, total energy consumption.
\end{abstract}

\section{Keywords}

Packet delivery ratio, packet delivery delay, control overhead, total energy consumption.

\section{INTRODUCTION}

A Mobile Ad hoc Network (MANET) is a fixed without infrastructure network, which is a collection of mobile devices and is self configuring, vigorously changing, multi-hop wireless network which forms a network via multi hop wireless network connection, it is a self-organizing network, without any central control. Each hub in a MANET must have the capacity to capacity to route and forward data to other nodes. When mobile applications must send the same information to more than one destination, multicasting is regularly utilized. Multicasting lessens the correspondence costs for applications that send the same information to various beneficiaries. As opposed to sending data by means of numerous unicasts, multicasting minimizes the connection transmission capacity utilization, switch transforming and conveyance delay. Existing multicast steering protocols for MANETs can be extensively characterized into tree-based directing conventions [1][2][3][4][5][6][7][8][9] and lattice based steering conventions [10][11][12][13][14][15].A tree structure that unites all multicast individuals and give one way between a couple of source and destination hubs using Treebased routing protocols. Mesh-based conventions yield a multi-way between the source and the destination hubs. At the point when a connection falls flat, work based multicast conventions don't have to re-process a cross sections.

Subsequently work based multicast conventions have a high bundle conveyance proportion contrasted with tree-based conventions, however they bring about more control and system overhead by flooding through the lattice. Royer and Perkins [7] proposed a multicast specially appointed on interest separation vector directing convention (MAODV). This protocol establishes on-demand multicast tree and use these for delivery of multicast data. For tree-based multicast routing we use MAODV is a typical protocols. The source and the destination nodes are connected through Mesh-based protocols yield a multi-path hoping between them. When a connection comes up short, work based multicast conventions don't have to re-process a cross sections.

In order to minimize the total transmission power in MANETs, routing protocols have been proposed to increase the lifetime of networks [16][17][18]. Minimum total transmission power (MTPR) establishes on-demand unicast routing based on the transmission power [17]. The MTPR routing scheme calculates the total transmission power for all routes between source and destination, then selects the route with minimum total transmission power among all routes to be the primary routing path. Minimum battery cost routing MBCR is similar to MTPR, MBCR finds the total battery cost for each route from source to destination and selects the route with minimum total remaining power [16]. MBCR prevents nodes from being abused and increases lifetime of networks. Wu and Tuan propose a power saving routing protocol with force sieving (PSRPS) [18]. PSRPS parts the system territory into a few square frameworks by the area data. Baolin and Layuan propose a reliable multicast routing protocol (dependability of the multicast impromptu on-interest separation vector (RMAODV)) by decreasing the quantity of course reproductions and bundle retransmissions [2].

In this paper, we study the Performance Evaluation and Comparison of PDTMRP and MAODV. In this scheme, we use the force mindful metric to get ahead of time the force utilization of transmitted information bundles. Then, the battery force is utilized to dispense with the temperamental hubs so as to attain to high unwavering quality. Two multicast trees are built to accomplish burden equalization. This paper is discussed in following number of section. Section 2 deals with Routing protocol. Section 3 Wireless Simulation, Section 4 deals with Simulation Results, Section 5 deals with Result Analysis, Section 6 deals with conclusion and Future Scope and section 7 deals with References.

\section{ROUTING PROTOCOL}

Ad hoc Routing protocol is classified into two types such as proactive and reactive. The table-driven routing protocol is proactive, it worked on distance vector based or link state based routing approaches. The disadvantage of this algorithm is the frequent updation is required which consumes large amount of memory, bandwidth and power [19].But, in the reactive routing protocol, each node does not need to maintain the routing table. When a source node is ready to send data, it initiates the route discovery procedure and maintains its routes 
only. The reactive routing protocol minimizes the routing overhead and also called on-demand approach.

\subsection{Multicast ad hoc on-demand distance vector routing protocol}

MAODV is a multicast extension for AODV protocol. MAODV based on shared trees on-demand to connect multicast group members. MAODV has capability of unicast, broadcast, and multicast. MAODV protocol can be route information obtained when searching for multicast; it can also increase unicast routing knowledge and vice-versa. When a node wishes to join a multicast group or it has data to send to the group but does not has a route to that group, it originates a route request (RREQ) message. Only the members of the multicast group respond to the join RREQ. If an intermediate node receives a join RREQ for a multicast group of which it is not a member or it receives a route RREQ and it doesn't have a course to that gathering, it rebroadcast the RREQ to its neighbors. But if the RREQ is not a join request any node of the multicast group may respond.

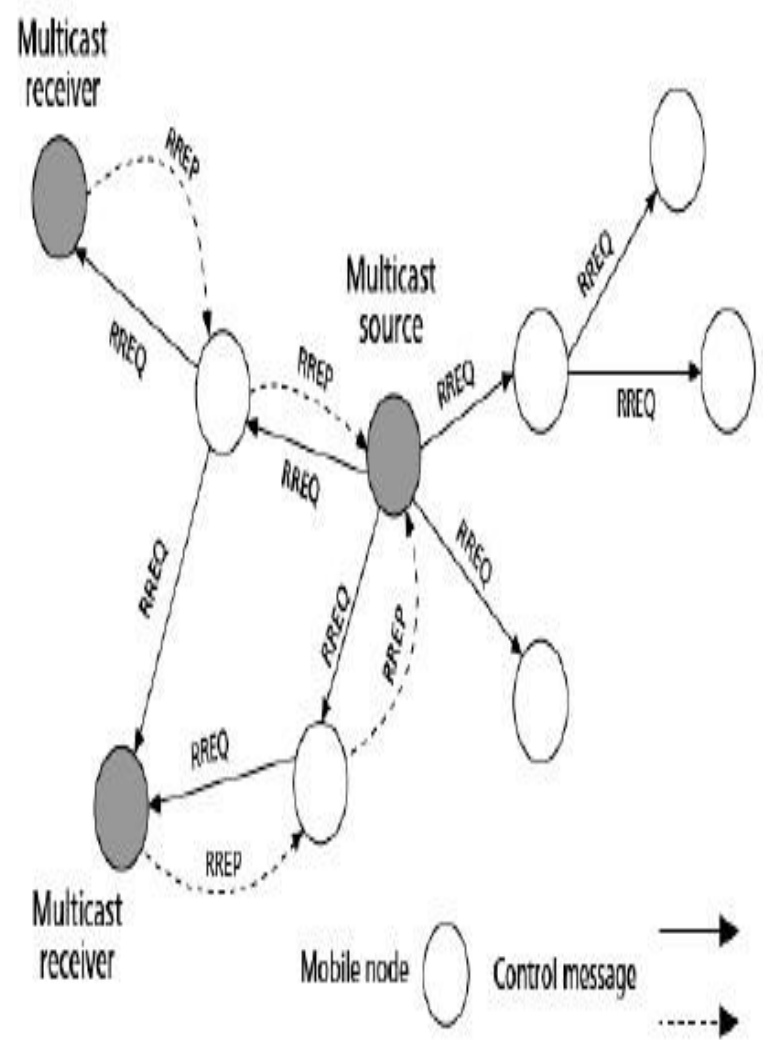

Fig 1: Path Discovery in the MAODV Protocol

\subsection{Power-aware dual-tree-based multicast routing protocol (PDTMRP)}

A Power-mindful double tree-based multicast directing convention (PDTMRP) for MANETs is proposed. In the proposed plan, all hubs are haphazardly ordered into two sorts, bunch 1 and gathering 2. To accomplish the heap adjust, two multicast trees (tree-1 for gathering 1 and tree-2 for group2) are built .Each hub kept up two steering tables: the neighboring table and the directing table. The neighboring table was easily obtained by the periodic broadcast of the hello packet. These tables are described below:

\subsubsection{Neighboring Table}

Any node which wants to know which are its neighbor with in its transmission range it will broadcast Hello packet. The nodes which are in transmission range will reply to Hello packet. The format of the table:

Table 1: To find the distance of neighboring node

\begin{tabular}{|l|l|}
\hline Nodeid & Distance \\
\hline
\end{tabular}

\subsubsection{Routing Table}

This table contained the path that was used for the transmission of data. The format of the path table was:

Table 2: To route the packet source to destination

\begin{tabular}{|l|l|l|l|l|}
\hline Source_Id & Dest_Id & Seqno & Routeclass & Next_hop \\
\hline
\end{tabular}

The Source_Id, Dest_Id fields contains the unique locations of the source and the destination hub, separately. The Seqno field contains the sequence number of the source node (guaranteeing the loop-freedom of all routes to the destination node). The course class field recorded the class of course for gathering 1 or gathering 2 . The Next hop field contained the location of the neighboring hub to which information parcels must be sent.
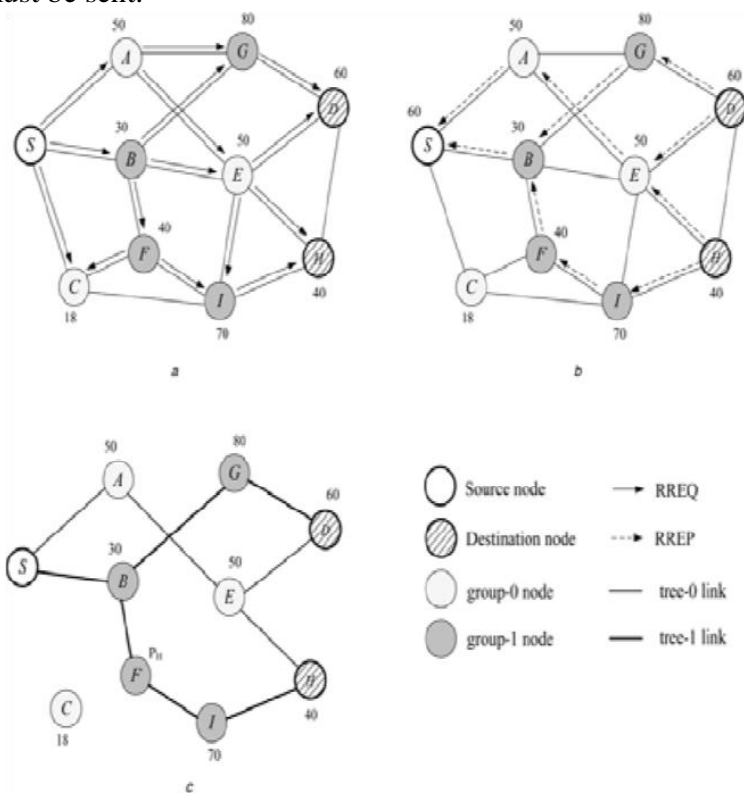

Fig 2: (a) shows route discovery process, (b) Shows route reply process and (c) Shows multicast trees. 


\subsection{Improvement in Network Lifespan by using Binary Tree Based Multicast Routing Protocol for Mobile Ad Hoc Network.}

In this study, an Enhanced Network Lifespan by tree-based multicast routing protocol (NLDTMRP) for MANETs is proposed. In the proposed arrangement, all hubs are haphazardly grouped into two sorts, aggregate 1 and gathering 2. To accomplish the heap adjust, two multicast trees (tree-1 for gathering 1 and tree-2 for group2) are built .Each hub kept up two steering tables: the neighbouring table and the routing table.

Neighboring Table: Any node which wants to know which are its neighbor with in its transmission range it will broadcast Hello packet. The destination node within the radius of that network will reply to hello packet.

Routing Table: The routing table contains some field which is used for transmission of data from the source node to destination node.

\subsubsection{Route Discovery Process}

In the proposed plan, force level edge (Pthreshold) is defined. When the source node wants to send the bundle to the destination hubs, it broadcasts the route request (RREQ) packet to the neighbouring hubs in its transmission range, when the source hub does not have a way in the directing table. The RREQ packet carries the following information in its header:

Table 3: To find all nodes in a network

\begin{tabular}{|c|c|c|c|c|c|c|}
\hline $\begin{array}{c}\text { TYP } \\
\text { E }\end{array}$ & Source & Des & Source & Path & Class & RRE \\
& & $\mathrm{t}$ & Seq & Traverse & & Q \\
& & List & & d & & Type \\
\hline
\end{tabular}

'Type' refers to the packet type: RREQ, RREP or RERR. 'Source' is the source node. 'SourceSeq' is a monotonically increasing sequence number. 'Source' and 'SourceSeq' are used to uniquely identify each RREQ packet. It can be used to check duplicate copies of an old request and detect the stale cached routes. 'DestList' is a set of destinations. 'Path Traversed' records the routing information. „Class is the type of node: group-1 or group-2. „RREQType refers to the RREQ type: RREQ, after neighboring nodes receive the RREQ packet, the neighbouring nodes first check the remaining battery of hubs (Premain). At the point when Premain of hubs is higher than $\mathrm{P}_{\text {threshold, }}$ the neighbouring hubs store got the RREQ bundle and re-showed the RREQ parcel. The neighbouring node adds its ID to the directing way field of the RREQ bundle and the class field of the RREQ bundle is assigned a type (group-1 or group-2) of neighbouring node. When the destination node receives the main RREQ bundle with gathering 1 and the principal RREQ bundle with gathering 2, the destination hub selects the last jump of every RREQ parcel as its upstream hub to be the essential directing ways for tree- 0 and tree- 1 . At that point, the destination hub sent two course answer (RREP) bundles to the source hub. The RREP packet carries the following information in its header:
Table 4: Sending a request packet from source

\begin{tabular}{|c|c|c|c|c|c|c|}
\hline TYPE & Source & Destination & $\begin{array}{c}\text { Source } \\
\text { Seq }\end{array}$ & $\begin{array}{c}\text { Reverse } \\
\text { path }\end{array}$ & Class & $\begin{array}{c}\text { RREP } \\
\text { Type }\end{array}$ \\
\hline
\end{tabular}

Here 'Type' is certainly RREP. 'Source' is the source node. 'Destination' is the destination node. The field 'ReversePath' in each RREP packet includes the reverse path. 'Class' is the type of node: group-1 or group-2.The Class field of RREP packet is the alloted sort for the RREQ bundle. At the point when the halfway hub receives the RREP packet, it selects the upstream hub taking into account the comparing sort of RREP bundle and sends the RREP bundles to the source hub. The point of interest of the course disclosure process.

\subsubsection{Algorithm 1: Route discovery process}

A system is demonstrated as chart $\mathrm{G}(\mathrm{N}, \mathrm{E})$, where $\mathrm{N}$ is the limited situated of portable hubs and $\mathrm{E}$ is a situated of connections. Assume $\mathrm{n}$ is the quantity of versatile hubs and $\mathrm{N}$ is the situated of portable hubs $\mathrm{N}=\{\mathrm{N} 1, \mathrm{~N} 2, \mathrm{Nn}\}$. Assume that source hub Ni needs to discover a way to destination hub $\mathrm{Nj}$. Hub Ni telecasts a RREQ parcel, and Nk gets the RREQ bundle, where $\mathrm{Ni}, \mathrm{Nj}, \mathrm{Nk}[\mathrm{N}, 1 \leq \mathrm{i}, \mathrm{j}, \mathrm{k} \leq \mathrm{n}$ and $\mathrm{i}=\mathrm{j}]$.

if (node $\mathrm{Nk}$ is the destination node $\mathrm{Nj}$ )

\{

1. Node Nk selects the first RREQ packet with group-1 and RREQ with group-2 as the upstream node and unicast a RREP packet to the source node.

2. Each node receives the reply RREP packet and writes the entry to the current routing table. Then the node selects an upstream node with a corresponding type of RREP.

\}

else if $\left(\mathrm{P}_{\text {remain }}\right.$ of node $\mathrm{Nk}$ is higher than $\left.\mathrm{P}_{\text {threshold }}\right)$

\{

1. Node Nk stores the received RREQ packet in its list of upstream nodes.

2. Node $\mathrm{Nk}$ forwards the RREQ packet to the neighbouring nodes.

\}

else

Node Nk discards the request packet.

\subsubsection{Route Maintenance Process}

It is divided in to three parts:

\subsubsection{Join Operation}

At the point when another part needs to join the multicast tree, it broadcasts a join route request (RREQJ) bundle over the systems. Just a hub that is an individual from the multicast tree (i.e. a switch for the gathering) may react, if a hub gets a RREQJ packet for a multicast group of which it is not a part or it doesn't have a course to that gathering, it makes an opposite course entrance to the forthcoming hub and afterward telecasts the RREQJ packet to its neighbours. Any intermediate node receives the RREQJ, it rebroadcasts the RREQJ on the off chance that the Premain of the hub is higher than $\mathrm{P}_{\text {threshold }}$. At the point when every part hub of the multicast tree gets the RREQJ packets it sends back the join route reply (RREPJ) parcel with set class field. At the point when every moderate 
hub gets the RREPJ parcel, the halfway hub chooses a downstream hub in view of the relating kind of RREPJ packet. The prospective node selects the first RREPJ parcel with gathering 1 and the first join answer with gathering 2 to join the multicast tree.

\subsubsection{Node Prune Operation}

At the point when a hub needs to move from the multicast tree, the pruning hub shows to its upstream hub a pruning route request (RREQP) packet. When the upstream node receives the RREQP packet, it expels the comparing entrance from its multicast directing table. In the event that the upstream hub turns into a leaf hub and it is not the tree collector, the hub can further prune itself from the tree.

\subsubsection{Broken Link Maintenance}

In PDTMRP, when a hub neglects to convey the information parcel to the following jump of the course, it considers the connection to be broken and sends a course lapse (RERR) bundle to the source hub. At the point when the upstream hub gets the RERR parcel, it expels the comparing entrance from its directing table and advances the RERR bundle to the source hub. If one of the two courses is broken, the source utilizes the staying substantial course to convey information bundles. At the point when both courses of the way are broken, the source hub starts the course revelation process. The RERR packet carries the following information in its header: <TYPE, Source, Destination, and Class $>$ Here 'Type' is certainly RERR. 'Source' is the source node. 'Destination' is the destination node. The field 'ReversePath' in each RREP packet includes the reverse path. 'Class' is the type of node: group- 0 or group-1.The course upkeep operation is demonstrated in Algorithm2

\subsubsection{Aglorithm2: Route Maintenance Process}

A network is modeled as graph $\mathrm{G}=(\mathrm{N}, \mathrm{E})$, where $\mathrm{N}$ is the finite set of mobile nodes and $\mathrm{E}$ is a set of links. Suppose $\mathrm{n}$ is the number of mobile nodes and $\mathrm{N}$ is the set of mobile nodes $\mathrm{N}$ $=\{\mathrm{N} 1, \mathrm{~N} 2, \ldots \mathrm{Nn}\}$. Assume that node $\mathrm{Ni}$ wants to send a packet to node $\mathrm{Nj}$, where $\mathrm{Ni}, \mathrm{Nj},[\mathrm{N}, 1<=\mathrm{i}, \mathrm{j}<=\mathrm{n}, \mathrm{i}=\mathrm{j}]$ and that the link between node $\mathrm{Ni}$ and node $\mathrm{Nj}$ breaks. if (the link of node $\mathrm{Ni}$ to node $\mathrm{Nj}$ breaks)

1. Node Ni saves the current data packet.

2. Node Ni broadcasts a repair route request (RREQR) packet to node $\mathrm{Nj}$, counts down $\mathrm{T}_{\text {time }}$ out seconds, and waits for the repair route reply (RREPR) packet to return.

If (the RREPR packet is back in $\mathrm{T}_{\text {time }}$ out seconds)

\{

1. Node Ni uses the replacement path to replace the path that breaks.

2. Node Ni continues packet transmission. \}

Else

Node Ni sends an RERR packet to the source and restarts the routing discovery process.

The simulation was implemented by using NS2 (Network Simulation 2, version 2.35) [20]. The simulation modeled a network in a $900 \mathrm{~m} \times 900 \mathrm{~m}$ area with varying mobile speed. We used random waypoint model was used as mobility model. In random waypoint model, each node randomly selects the moving direction, and when it reaches to the boundary of simulation area, it bounces back and continues to move. The transmission extent was $150 \mathrm{~m}$. The information parcel size was 250 bytes. The initial power of each node was $10 \mathrm{~J}$. Pthreshold was $0.5 \mathrm{~J}$. Each simulation was executed for $600 \mathrm{sec}$. The source and destination hubs were arbitrarily picked and every hub was haphazardly relegated an introductory vitality. We used constant bit rate (CBR) as the traffic type. In CBR model, the source transmits a certain number of fixed size packets. The parameters utilized as a part of the reproductions are recorded in table 5 .

Table 5: Simulation Parameters

\begin{tabular}{|c|c|}
\hline Parameter & Values \\
\hline Examined protocols & MAODV, PDTMRP \\
\hline Simulation Time & $600 \mathrm{sec}$ \\
\hline Number of Nodes & 100 \\
\hline mobility speed & $1-25 \mathrm{~m} / \mathrm{s}$ \\
\hline mobility model & Random way point model \\
\hline $\begin{array}{c}\text { Simulation Area } \\
\text { node transmission } \\
\text { range }\end{array}$ & $900 \times 900$ \\
\hline data packet size & $150 \mathrm{~m}$ \\
\hline Traffic Type & 250 bytes \\
\hline
\end{tabular}

The execution assessment measurements utilized as a part of the:

1. Packet Delivery Ratio: - The data packets delivered divided by the data packets expected to be delivered.

2. Control Overhead:-The control packets transmitted divided by the data packets delivered.

3. Packet Delivery Delay: - The interval from the time the multicast is initiated to the time the last host finishes its multicasting.

4. Total Energy Consumption: - The total consumed Energy of all nodes after data transmission.

\section{WIRELESS SIMULATION}

The Network simulation-2 implementation has following important parts.

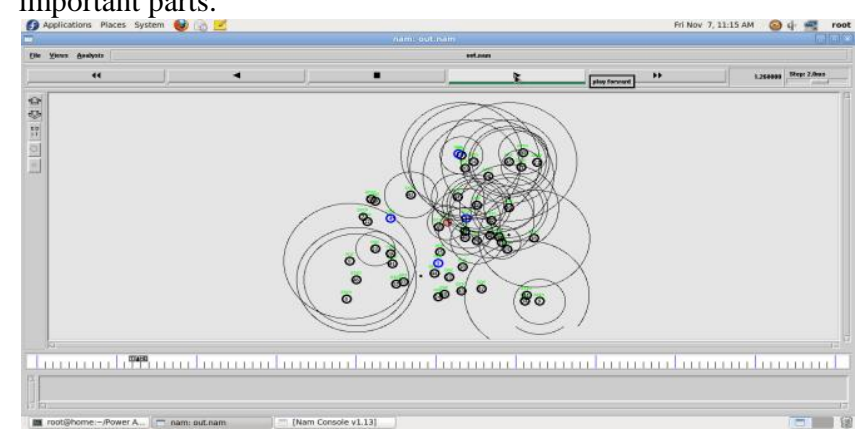

Fig 3: Simulation shown with NAM animator 
1. Generating wireless Environment.

2. Creating UPD and FTP Agent.

3. Various modules are added to simulate node mobility and wireless networking such as mobile node, ad-hoc routing such as aodv, MAC802.1.

4. Radio propagation Model and channel etc.

Nam is a Tcl/TK based movement device for review system reenactment follows and true bundle follow data. It bolsters topology format, bundle level activity, and different information investigation instruments. The simulation was implemented by using NS2 (Network Simulation 2, version 2.35) [20]. The simulation modeled a network in a $900 \mathrm{~m} \times$ $900 \mathrm{~m}$ area with 50 mobile nodes. We used random way point model was used as mobility model. In random waypoint model, each node randomly selects the moving direction, and when it reaches to the boundary of simulation area, it bounces back and continues to move. The portable rate of every hub was from 1 to $25 \mathrm{~m} / \mathrm{s}$. The transmission extent was $150 \mathrm{~m}$. The information parcel size was 250 bytes. The initial energy of each node was $10 \mathrm{~J}$. P (threshold) was $0.15 \mathrm{~J}$. Each simulation was executed for $600 \mathrm{~s}$. The value in the following simulation figures are the average values of 50 runs. The source and destination hubs were haphazardly picked and each node as randomly assigned an initial energy. We used constant bit rate $(\mathrm{CBR})$ as the traffic type.

\section{SIMULATION RESULTS}

In the accompanying, the effect of versatility speed on MAODV, PDTMRP is studied. These protocols have been simulated for packet delivery ratio, packet delivery delay and total energy consumption, control overhead.

From Figs. 4-7, we depict the routing performance of two protocols under different mobility speeds. Fig. 4 demonstrates the execution of the bundle conveyance proportion under different portability speeds. As indicated in Fig.4, the bundle conveyance proportion diminished with expanding portability because of more link breaks. Notice that the packet delivery ratio is high when the nodes have low mobility. PDTMRP achieves a much higher packet delivery ratio than MAODV because energy is assessed while securing of two steady steering ways for multicasting. Hence, the parcel conveyance proportion of PDTMRP is higher than that of MAODV.

From Fig.5 we depict performance of the packet delivery delay under different versatility speeds. As demonstrated in Fig.5, as the mobility speed increases, the packet delivery delay also increases. The packet delivery delay of PDTMRP is lower MAODV. This is also because vitality is assessed while making of two steady directing ways for multicasting.

From Fig. 6, shows the performance of the total energy consumption energy under various mobility speeds. Inferable from the versatility of the hub making the control overhead increases, it consumes more energy. Therefore the total energy consumption increases with expanding portability. As saw in Fig. 6, the total energy consumption of PDTMRP is lower than that of MAODV. This is because of PDTMRP reducing the energy consumption by using dual trees for transmission.

Fig. 7 demonstrates the execution of the control overhead under different portability speeds. As is expected, the control overhead increments as the versatile hubs got to be more portable. The reason is that there are more risks for courses to break when the rate of the portable hubs is faster. Thus, the number of rebroadcasts increased. PDTMRP not only eliminates wasteful hubs to diminishing the quantity of control parcels, additionally structures double trees to diminish the quantity of course recreations. In this manner PDTMRP has a lower control overhead than MAODV.

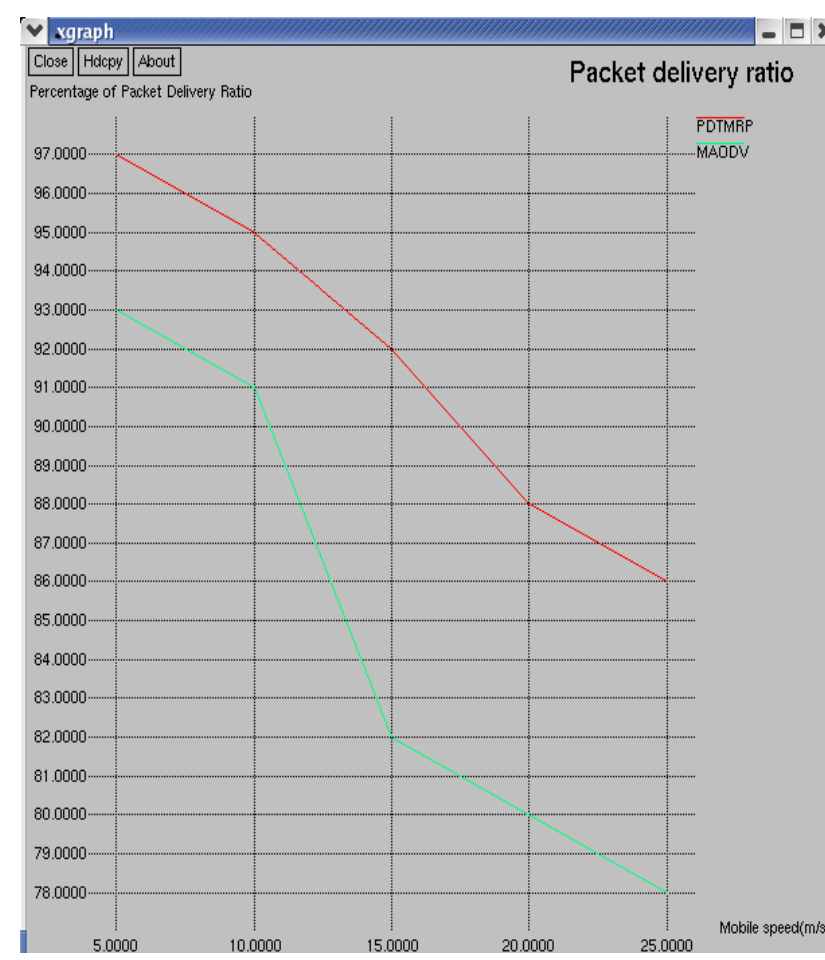

Fig 4: Packet delivery ratio against mobility speed

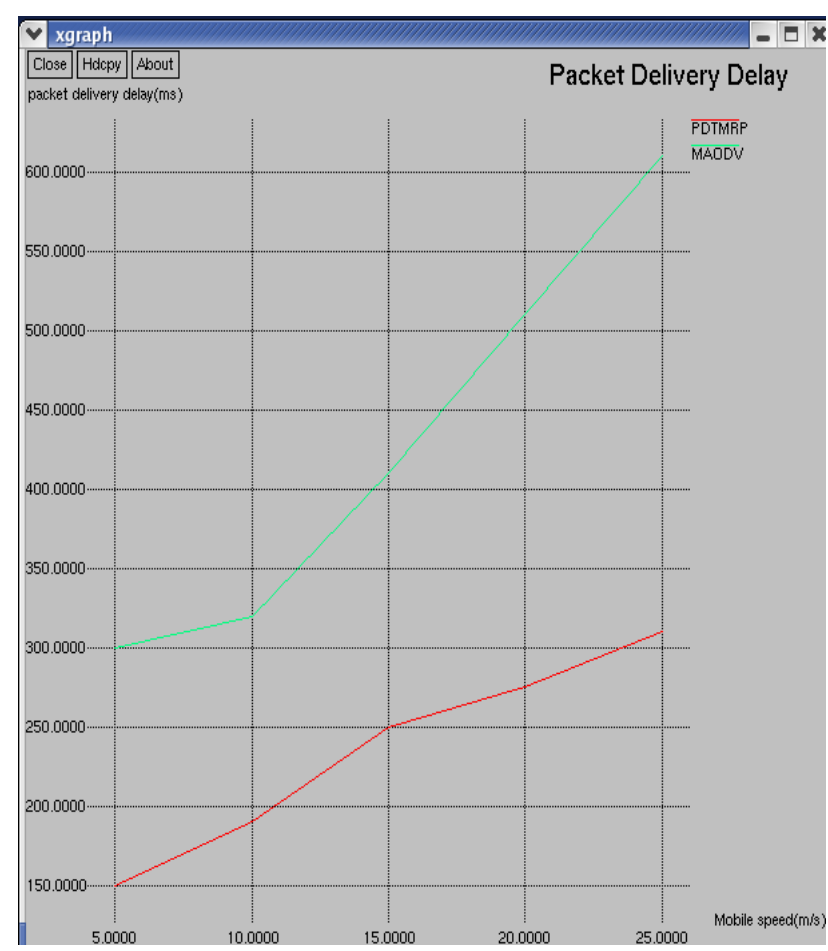

Fig 5: Packet delivery delay against mobility speed 


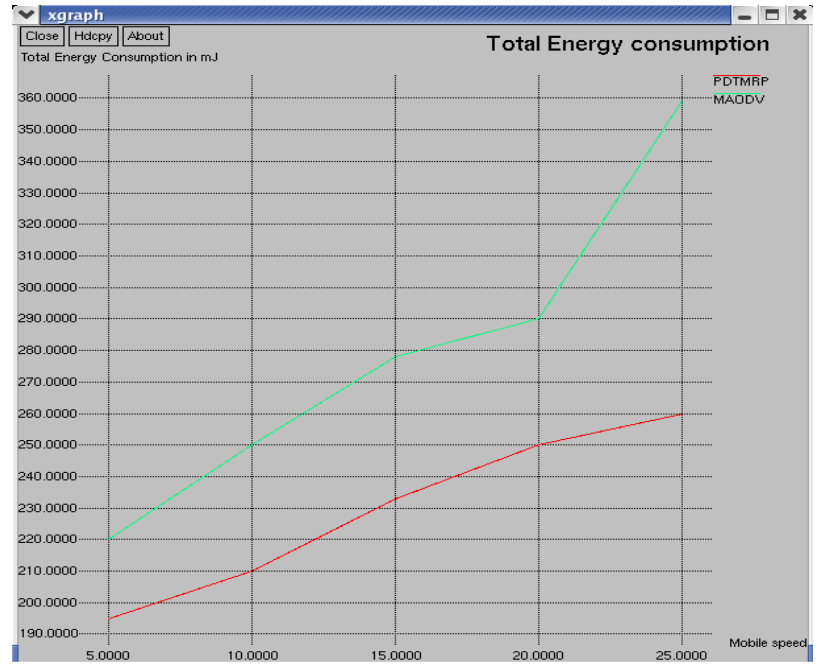

Fig 6: Total Energy consumption against mobility speed

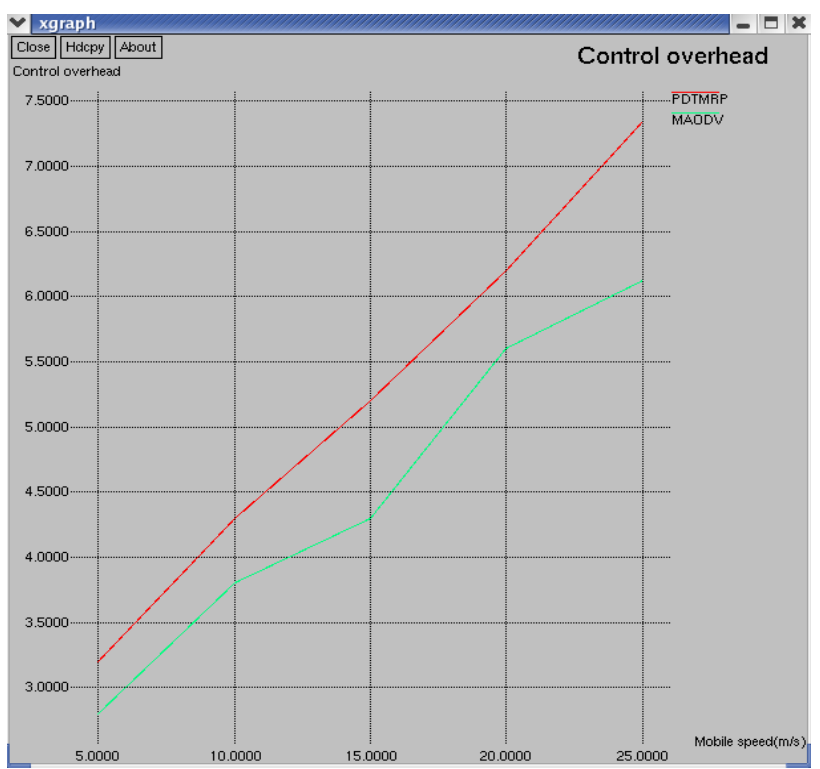

Fig 7: Control overhead against mobility speed

\section{RESULT ANALYSIS}

Table 6: Packet Delivery Ratio

\begin{tabular}{|c|c|c|}
\hline & PDTMRP & MAODV \\
\hline 50000 & 970000 & 930000 \\
\hline 100000 & 950000 & 910000 \\
\hline 150000 & 920000 & 820000 \\
\hline 200000 & 880000 & 800000 \\
\hline 250000 & 860000 & 780000 \\
\hline
\end{tabular}

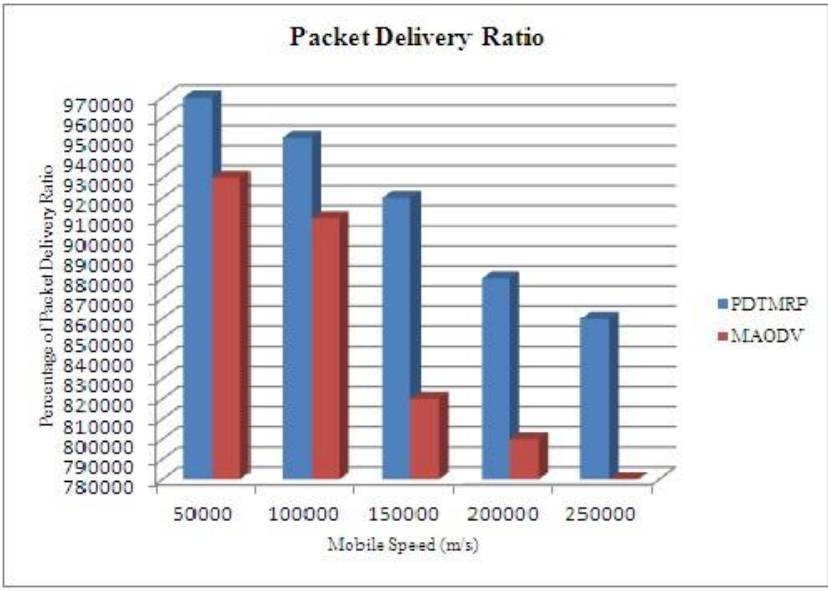

Table 7: Packet Delivery Delay

\begin{tabular}{|c|c|c|}
\hline & PDTMRP & MAODV \\
\hline 50000 & 1500000 & 3000000 \\
\hline 100000 & 1900000 & 3200000 \\
\hline 150000 & 2500000 & 4100000 \\
\hline 200000 & 2750000 & 5100000 \\
\hline 250000 & 3100000 & 6100000 \\
\hline
\end{tabular}

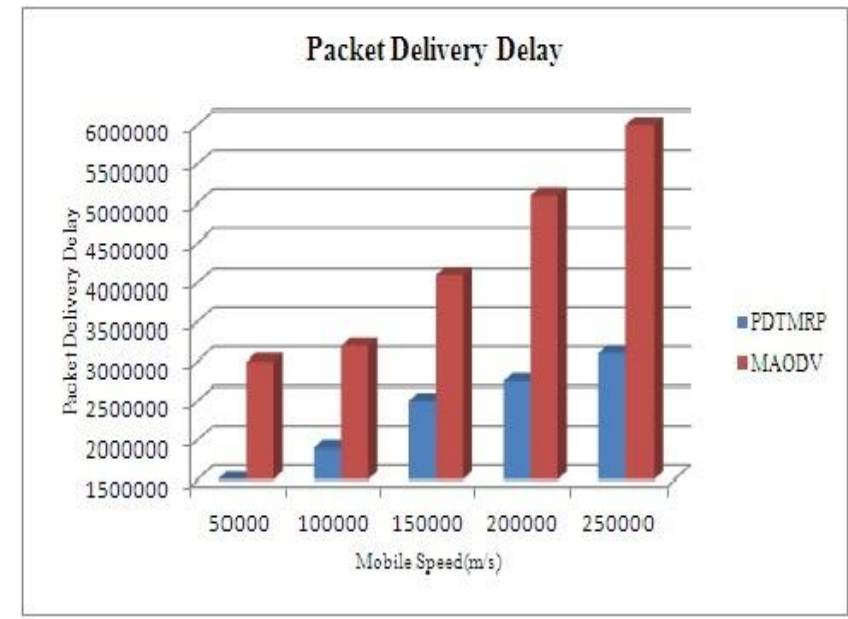

Table 8: Total Energy Consumption

\begin{tabular}{|c|c|c|}
\hline & PDTMRP & MAODV \\
\hline 50000 & 1950000 & 2200000 \\
\hline 100000 & 2100000 & 2500000 \\
\hline 150000 & 2320000 & 2790000 \\
\hline 200000 & 2500000 & 2900000 \\
\hline 250000 & 2600000 & 3600000 \\
\hline
\end{tabular}




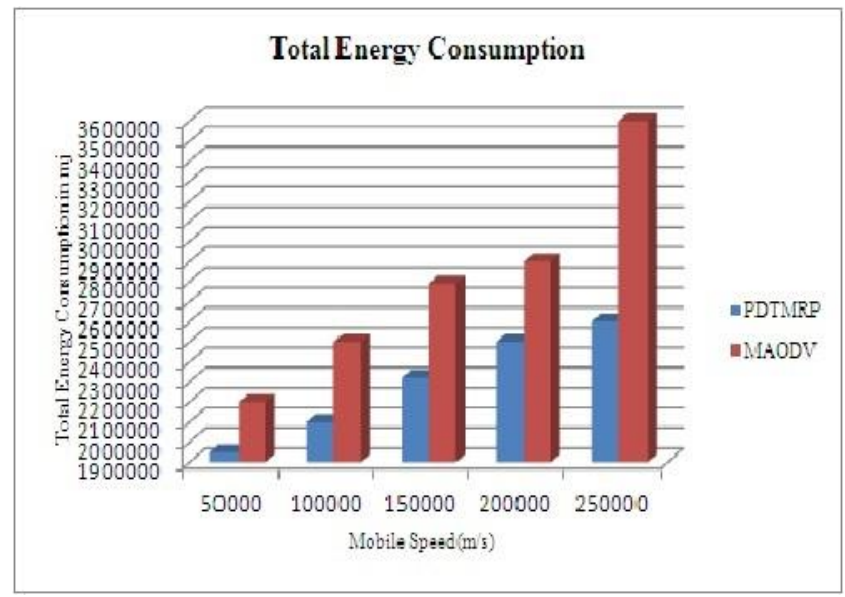

Table 9: Control Overhead

\begin{tabular}{|c|c|c|}
\hline & PDTMRP & MAODV \\
\hline 50000 & 3.3000 & 2.7500 \\
\hline 100000 & 4.2500 & 3.7500 \\
\hline 150000 & 5.2300 & 4.3000 \\
\hline 200000 & 6.2300 & 5.6000 \\
\hline 250000 & 7.3500 & 6.1000 \\
\hline
\end{tabular}

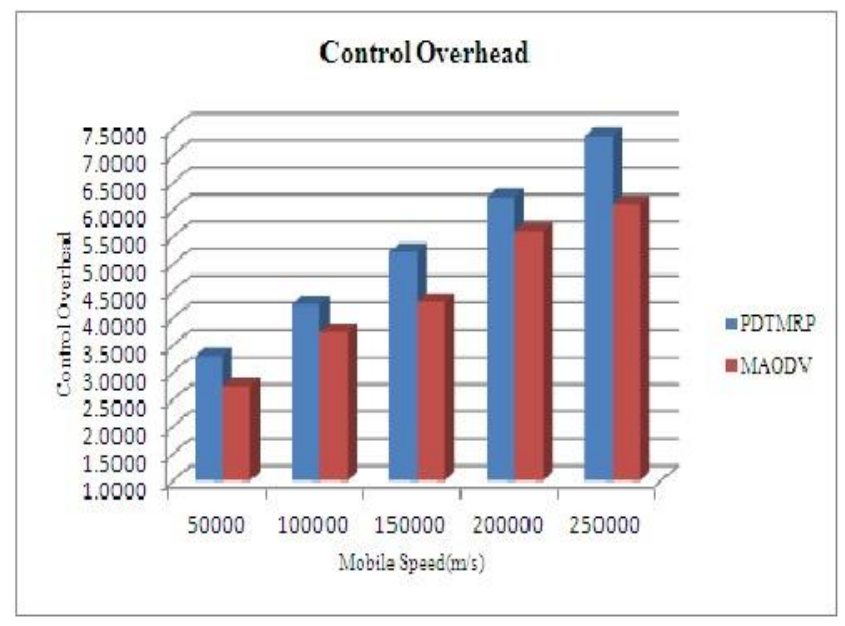

\section{CONCLUSION AND FUTURE SCOPE}

In this paper, we design a PDTMRP for MANETs. The PDTMRP improves load balance is used to increase the lifetime of a system. In the course discovery, the PDTMRP not only improves the route stability of multicast routing, be that as it may, additionally attains to the heap offset of information transmission. Accordingly the control overhead for course development and the quantity of course recreations can be diminished. Reenactment results demonstrate that the parcel conveyance proportion and the packet delivery delay of the PDTMRP outperform that of MAODV protocol. PDTMRP is an energy-aware multicast routing protocol. The node with low energy does not select as a member of multicast tree. PDTMRP improves the route stability of multicast routing. The total energy consumption can be decreased and the network lifetime can be prolonged. Improvement for proposed convention should be possible by utilizing binary or ternary characterizing hubs as a part of to gathering 1 , gathering 2 , and gathering 3 .We can amplify the proposed framework utilizing interchange way directing way. Several other evaluations measures can be added to the checkout various comparison metrics. The nature of the graph can be altered for better understanding.

\section{REFERENCES}

[1] Ballaradie, A., Crowcroft, J., Francis, P.: 'Core based tree (CBT) - an architecture for scalable interdomain multicast routing protocol'. Proc. ACM SIGCOM, October 1993, pp. 85-89.

[2] Baolin, S., Layuan, L.: 'On the reliability of MAODV in ad hoc networks'. Proc. IEEE Int. Symp. On Microwave, Antenna, Propagation and EMC Technologies for Wireless Communications, August 2005, vol. 2, pp. 1514-1517.

[3] Bommaiah, E., Liu, M., McAuley, A., Talpade, R.: 'AMRoute: ad hoc multicast routing protocol'. Internet Draft, August 1998.

[4] Calvert, K.L., Zegura, E.W., Donahoo, M.J.: 'Core selection methods for multicast routing'. Proc.

Fourth Int. Conf. on Computer Communications and Networks, September 1995, pp. 638-642.

[5] Das, S.K., Manoj, B.S., Murthy, and C.S.R.: 'A dynamic core based multicast routing protocol for ad hoc wireless network'. Proc. Third CM Int. Symp. On Mobile Ad Hoc Networking and Computing, 2002, pp. 24-35.

[6] Macker, J.P., Corson, M.S.: 'Mobile ad hoc networking and the IETF', ACM SIGMOBILE Mob. Comput. Commun. Rev.., 1998, 2, (2), pp. 9-14.

[7] Royer, E.M., Perkins, and C.E.: 'Multicast operation of the ad hoc on demand distance vector routing protocol'. ACM MOBICOM, 1999, pp. 207-218.

[8] Wang, B., Gupta, S.K.S.: 'On maximizing lifetime of multicast tree in wireless ad-hoc networks'. Proc. Int. Conf. on Parallel Processing, October 2003, pp. 333-340.

[9] Wu, C.W., Tay, Y.C., Toh, C.K.: 'Ad hoc multicast routing protocol utilizing increasing ID-numbers (amris) functional specification'.Internet Draft, November 1998.

[10] Chiang, C., Gerla, M., Zhang, L.: 'Forwarding group multicast protocol (FGMP) for multihop, mobile wireless networks', Cluster Comput., Spec. Issue Mob. Comput. 1998, 1, (2), pp. 187-196.

[11] Devarapalli, V., Sidhu, D.: 'MZR: A multicast protocol for mobile ad hoc networks'. Proc. IEEE Int. Conf. on Communications, June 2001, pp. 886-891.

[12] Garcia-Luna-Aceves, J.J., Madruga, E.L.: 'The coreassisted mesh protocol', IEEE J. Sel. Areas Commun., 1997, 17, (8), pp. 1380-1394.

[13] Gui, C., Mohapatra, P.: 'Efficient overlay multicast for mobile ad hoc networks'. Proc. IEEE Wireless Communication and Networking Conf., 2003, 2, pp. $1118-1123$. 
[14] Lee, S.J., Gerla, M., Chiang, C.C.: 'On demand multicast routing protocol'. Proc. IEEE Wireless Communication and Networking Conf., September 1999, pp. 1298-1302.

[15] Sinha, P., Sivakumar,R.,Bharghavan, V.:'MCEDAR: multicast core extraction distributed ad- hoc routing'. Proc. Wireless Communications and Networking Conf., September 1999, pp. 1313-1317.

[16] Singh, S., Woo, M., Raghavendra, C.S.: 'Energy- aware routing in mobile ad hoc networks'. Proc. Fourth Annual ACM/IEEE Int. Conf. on Mobile Computing and Networking, October 1998, pp. 181 181-190.

[17] Toh, C.K., Cobb, H., Scott, D.A.: 'Performance evaluation of battery life-aware routing scheme for wireless ad hoc networks'. Proc. IEEE Int. Conf. on Communications, 2001, vol. 9, pp. 2824-2829.
[18] Wu, Y.-C., Tuan, C.-C.: 'Energy saving routing protocol with energy sieving in wireless ad hoc networks'. Proc. Int. Conf. on Networks Security, Wireless Communications and Trusted Computing, April 2009, vol. 1, pp. 349-352.

[19] C. Sivaram murthy and B.S. Manoj, Adhoc Wireless Networks Architectures and Protocols, Pearson Education, 2nd ed., 2005.

[20] Guru Prasad, Prof Arvind S "Energy Optimization and Load Balancing using Tree Based Multicast Routing Protocol for Mobile Adhoc Network" International Journal of Engineering Research \& Technology (IJERT) 8 Vol. 2 Issue 6, June - 2013. 